\title{
Bone morphology allows estimation of loading history in a murine model of bone adaptation
}

\author{
Patrik Christen - Bert van Rietbergen • \\ Floor M. Lambers • Ralph Müller • Keita Ito
}

Received: 30 January 2011 / Accepted: 20 June 2011 / Published online: 7 July 2011

(C) The Author(s) 2011. This article is published with open access at Springerlink.com

\begin{abstract}
Bone adapts its morphology (density/microarchitecture) in response to the local loading conditions in such a way that a uniform tissue loading is achieved ('Wolff's law'). This paradigm has been used as a basis for bone remodeling simulations to predict the formation and adaptation of trabecular bone. However, in order to predict bone architectural changes in patients, the physiological external loading conditions, to which the bone was adapted, need to be determined. In the present study, we developed a novel bone loading estimation method to predict such external loading conditions by calculating the loading history that produces the most uniform bone tissue loading. We applied this method
\end{abstract}

\section{P. Christen}

Orthopaedic Biomechanics, Department of Biomedical

Engineering, Eindhoven University of Technology, PO Box 513,

WH 4.11, 5600 MB, Eindhoven, The Netherlands

e-mail: p.christen@tue.nl

B. van Rietbergen $(\varangle)$

Orthopaedic Biomechanics, Department of Biomedical

Engineering, Eindhoven University of Technology, PO Box 513,

WH 4.128, $5600 \mathrm{MB}$, Eindhoven, The Netherlands

e-mail: b.v.rietbergen@tue.nl

\section{F. M. Lambers}

Institute for Biomechanics, ETH Zurich, HCI E 451,

Wolfgang-Pauli-Str. 10, 8093 Zurich, Switzerland

e-mail: flambers@ethz.ch

\section{R. Müller}

Institute for Biomechanics, ETH Zurich, HCI E 357.2,

Wolfgang-Pauli-Str. 10, 8093 Zurich, Switzerland

e-mail: ram@ethz.ch

\section{K. Ito}

Orthopaedic Biomechanics, Department of Biomedical Engineering, Eindhoven University of Technology, PO Box 513, WH 3.115,

$5600 \mathrm{MB}$, Eindhoven, The Netherlands

e-mail: k.ito@tue.nl to murine caudal vertebrae of two groups that were in vivo loaded by either 0 or $8 \mathrm{~N}$, respectively. Plausible load cases were sequentially applied to micro-finite element models of the mice vertebrae, and scaling factors were calculated for each load case to derive the most uniform tissue strainenergy density when all scaled load cases are applied simultaneously. The bone loading estimation method was able to predict the difference in loading history of the two groups and the correct load magnitude for the loaded group. This result suggests that the bone loading history can be estimated from its morphology and that such a method could be useful for predicting the loading history for bone remodeling studies or at sites where measurements are difficult, as in bone in vivo or fossil bones.

Keywords Bone loading estimation - Bone adaptation . Micro-finite element analysis - Bone remodeling algorithm • Murine caudal vertebra

\section{Introduction}

Trabecular bone can display a wide variety of architectures. It is well known that these different architectures are related to the load-carrying function of the bone. Sites at which the loading direction varies little, e.g., vertebrae, show a clear unidirectional rod-like architecture, whereas sites in which the loading directions vary over a wide range (e.g., hip and mandible) show more deviation and a more plate-like architecture. Such a plate-like structure is capable to withstand loads from different directions, as there is always a plate in the plane of the loading direction (Giesen and van Eijden 2000; Hildebrand et al. 1999). Load magnitude also plays an important role: High loads will result in a dense platelike architecture, whereas lower loads are likely to produce 
low-density rod-like structures (Ding et al. 2002; Whitehouse and Dyson 1974).

It is generally accepted that this relationship between bone morphology (density/micro-architecture) and loading is the result of a local load-adaptive bone remodeling process regulated and carried out by bone cells in such a way that bone tissue is added to high-load locations and removed from low-load locations ('Wolff's law') (Schulte et al. 2011; Adams et al. 1997; Forwood and Turner 1995; Goldstein et al. 1991; Rubin and Lanyon 1987; Frost 1987; Wolff 1892). This implies that, eventually, all bone tissue should be loaded uniform, thus producing a bone morphology that is optimized for the external loading history that it is subjected to. Since bone morphology is also influenced by other factors, e.g., calcium homeostasis, and morphogenesis, the tissue is not loaded perfectly uniformly. Although it is uncertain how uniformly a certain bone is loaded, a recent study shows that external forces clearly affect trabecular bone architecture besides predetermined factors (Abel and Macho 2011).

This causal relationship between bone loading history and morphology, governed by the bone remodeling process, suggests that it might be possible as well to derive the loading history from the actual micro-architecture and density. This can be done by finding the set of external forces that produces a uniform bone tissue loading, i.e., the inverse of 'Wolff's law'. Such an inverse procedure, however, can only be successful if distinct bone morphology is found for each corresponding load and if the assumption of uniform loading conditions is met reasonably well. By optimizing the magnitude of a set of 'plausible' external forces, it should then be possible to find the loading history to which the bone has adapted to over time. Presently, however, it is not known to what extent these premises are met.

If this approach indeed is feasible, it would be of great value for several applications. First, it would enable to estimate the bone loading history in cases where the bone morphology can be assessed (e.g., by micro-CT) but no loading conditions can be measured, such as for bone in vivo or fossil bones. Second, such an algorithm could derive the loading conditions needed to simulate bone remodeling for existing bone morphologies. Finding the forces to which the bone architecture and density are remodeled would enable to predict the course of bone remodeling when conditions are changing, thus enabling patient-specific predictions of bone morphology.

This concept was explored in earlier studies using continuum-level finite element models that accounted for the bone density only. Fischer et al. (1995) developed an approach in which the magnitude of a predefined set of 'plausible' loads is optimized for a uniform tissue stress throughout the bone. Since continuum models cannot model the bone architecture, the actual bone tissue stress could only be estimated from the continuum stress and bone density. Later, this approach was applied to the human proximal femur (Fischer et al. 1998, 1999), and it was shown that it is possible to determine dominant load cases that were generally in agreement with published experimental data for gait. At a more local level, a similar approach was successfully used to predict contact forces within a joint as a function of bone density (Bona et al. 2006). Although successful, these approaches were limited to $2 \mathrm{D}$ density-based tissue loading estimations and were not able to predict complex loading conditions.

With the development of 3D micro-finite element (microFE) analysis, it is now possible to represent the trabecular architecture and density in detail and to calculate the bone tissue-level stresses and strains generated by external forces. This enables the exploration of the concept that loading history can be determined from the bone morphology in a much more rigorous manner: including the full (anisotropic) bone architecture and the actual bone tissue-level stresses and strains. When combined with similar optimization procedures as used in these earlier studies, this makes it possible to calculate the magnitude of 'plausible' external loading conditions using uniform tissue loading as an objective function.

In this paper, we hypothesized that the bone loading history can be estimated from its architecture and density distribution when using micro-FE models for the calculation of bone tissue loading conditions. Specific goals of this study were twofold. First, to test whether the micro-FE-based approach is able to predict loading conditions for a set of test models and for two groups of mice that had their caudal vertebra loaded by either 0 or $8 \mathrm{~N}$, respectively. Second, to investigate to what extent homogeneity of bone tissue loading can be achieved when using a limited set of 'plausible' external forces.

\section{Materials and methods}

\subsection{Theory}

We assumed that bone adapts to a daily loading history that can be represented by a limited number of $n$ load cases $\vec{F}_{i}$ that are applied to the bone sequentially (Fig. 1). Each of these load cases $i$ has a specific load magnitude $F_{i}$ and is assumed to act $m_{i}$ times per day. During its application, the load case generates a specific state of bone tissue loading at point $x$ in the bone tissue, which is quantified here by the strain-energy density (SED) $U_{i}(x)$. During the daily loading history, a total of $m_{i}$ load cycles are applied, and the average SED value that is experienced at point $x$ in the bone tissue $\bar{U}(x)$ can then be described as:

$\bar{U}(x)=\sum_{i=1}^{n} \frac{m_{i}}{m_{\mathrm{tot}}} U_{i}(x)$ 


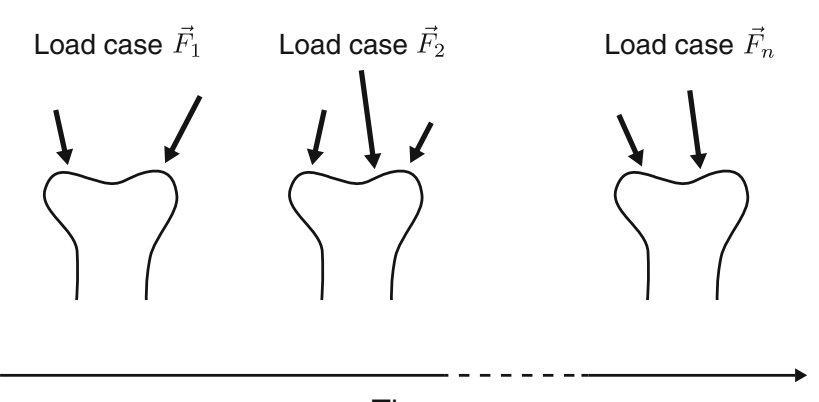

Time

Fig. 1 Typical daily loading history represented by a limited number of $n$ load cases $\vec{F}_{i}$ that are applied to the bone sequentially, where each arrow represents a load with its direction and magnitude

with $m_{\text {tot }}$ the total number of load cycles for all load cases.

For the present implementation, all load cases must be represented by one or more external forces that act on the bone at the same time in a specific direction and with a unit value. If the load case involves several forces with different magnitudes, the largest applied force should be scaled to a unit magnitude and the other forces should be scaled accordingly. The magnitude of the load case can then be scaled to that of the real situation by multiplying the unit load case by a factor $\alpha_{i}$ :

$\vec{F}_{i}=\alpha_{i} \vec{F}_{i_{\text {unit }}}$

For linear elastic behavior, the average $\operatorname{SED} \bar{U}(x)$ is dependent on the magnitude of the externally applied forces according to:

$\bar{U}(x)=\sum_{i=1}^{n}\left(\frac{m_{i}}{m_{\mathrm{tot}}} \alpha_{i}^{2}\right) U_{i_{\mathrm{unit}}}(x)=\sum_{i=1}^{n} s_{i} U_{i_{\text {unit }}}(x)$

with $U_{i_{\text {unit }}}(x)$ the local SED at point $x$ for unit load case $\vec{F}_{i_{\text {unit }}}$. The term $s_{i}=\frac{m_{i}}{m_{\text {tot }}} \alpha_{i}^{2}$ thus represents a scaling factor for the local SED values that depends on the loading duration and the magnitude of the load case.

With this set of definitions, the concept explored in this study implies finding the scaling factor $s_{i}$ that minimizes the following residual function $r\left(s_{i}\right)$ :

$$
\begin{aligned}
\min _{s_{i}} r\left(s_{i}\right) & =\int[\bar{U}(x)-k]^{2} \mathrm{~d} V \\
& =\int\left[\sum_{i=1}^{n}\left[s_{i} U_{i_{\text {unit }}}(x)\right]-k\right]^{2} \mathrm{~d} V
\end{aligned}
$$

with $k$ a target value for the local SED. In the present study, this target value was set to $0.02 \mathrm{MPa}$ (Mullender and Huiskes 1995).

It should be noted that the scaling factors represent the combined effect of the duration $\frac{m_{i}}{m_{\mathrm{tot}}}$ and magnitude $\alpha_{i}$ of a load case. However, if assumptions can be made about the number of cycles that a specific load case occurs relative to the total number of load cycles, it will be possible to derive the magnitude of the load case from:

$\alpha_{i}=\sqrt{\frac{m_{\mathrm{tot}}}{m_{i}} s_{i}}$

Alternatively, if the magnitude of a load case is known, it will be possible to derive its load duration from:

$\frac{m_{i}}{m_{\mathrm{tot}}}=\frac{s_{i}}{\alpha_{i}^{2}}$.

\subsection{Computational implementation}

After defining the 'plausible' load cases, micro-FE analyses are used to calculate the local SED values for each unit load case $U_{i_{\text {unit }}}(x)$. In the present study, a voxel conversion technique (van Rietbergen et al. 1995) is used to create the micro-FE models and the SED values are evaluated at the center of the elements. The SED values for each load case and each element are stored in a matrix $\mathbf{U}$, where the columns represent the load case $i$ and the rows the element $j$ of the micro-FE model. To exclude very highly loaded elements, e.g., due to loosely connected trabeculae, SED values greater than the 95 percentile are set to the value of the 95 percentile. Scaling matrix $\mathbf{U}$ with a scaling factor $s_{i}$, for each load case $i$, in such a way, that a bone tissue SED closest to a target value $k$ is obtained, when results for all scaled load cases are added, equation 4 can be formulated as:

$$
\min _{s_{i}} \mathbf{r}=\left\|\left[\begin{array}{ccc}
U_{1,1} & \ldots & U_{i, 1} \\
\vdots & \ddots & \vdots \\
U_{1, j} & \ldots & U_{i, j}
\end{array}\right]\left[\begin{array}{l}
s_{1} \\
\vdots \\
s_{i}
\end{array}\right]-k\left[\begin{array}{c}
1 \\
\vdots \\
1
\end{array}\right]\right\|^{2} V_{e}
$$

where $\|\cdots\|$ indicates the Euclidian norm, and $V_{e}$ represents the element volume. Since with the use of a voxel conversion technique all element volumes are the same, the element volume is a constant and can be omitted from the calculation. Because SED is a positive measure only, it is subjected to non-negativity constraints, leading to the following minimization problem formulation:

$$
\begin{gathered}
\min _{s_{i}} \mathbf{r}=\|\mathbf{U} \mathbf{s}-\mathbf{k}\|^{2} \\
\text { subject to } s_{i} \geq 0
\end{gathered}
$$

Scaling factors $s_{i}$ for each load case were then calculated using a non-negative linear least square optimization technique (MATLAB, The MathWorks Inc., Natick MA, USA). This is an active set method based on the algorithm described in Lawson and Hanson (1974). An active set refers to constraints with a negative or zero result $\left(s_{i}\right)$ when treated unconstraint. Otherwise, the set is passive. To find the active set, variables are identified and removed iteratively from the active set in such a way that the residuals decrease steadily. After a finite number of iterations, the true active set is found 
Fig. 2 Micro-FE models and their boundary conditions $(B C)$. For the grid models $(\mathbf{a}, \mathbf{b})$, the first three load cases represent a distributed normal force of $1 \mathrm{~N}$ in each of the three orthogonal directions and the last three load cases represent a distributed shear force in the three orthogonal directions. For the murine caudal vertebra model (c), the six unit load cases represent distributed unit forces $(1 \mathrm{~N})$ and distributed unit moments $(1 \mathrm{~N} \mathrm{~mm})$ in the three orthogonal directions applied to the end of the intervertebral disks (purple blocks) only a
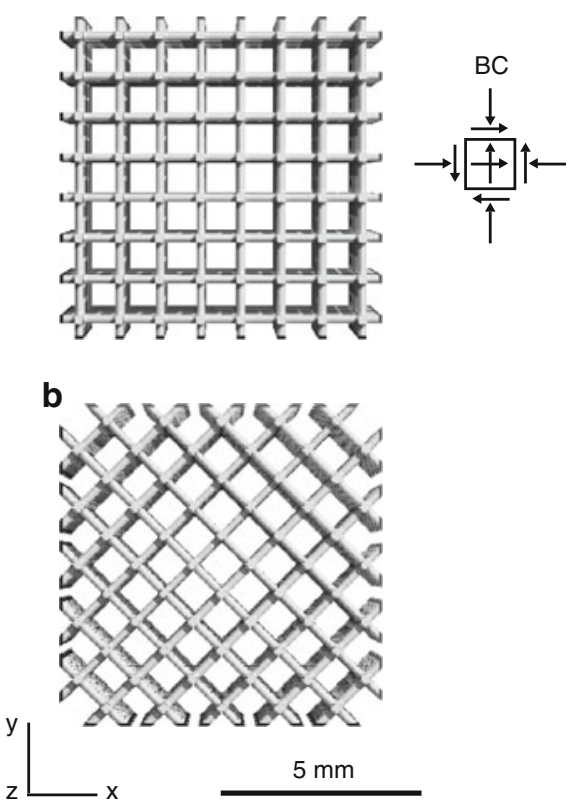

c

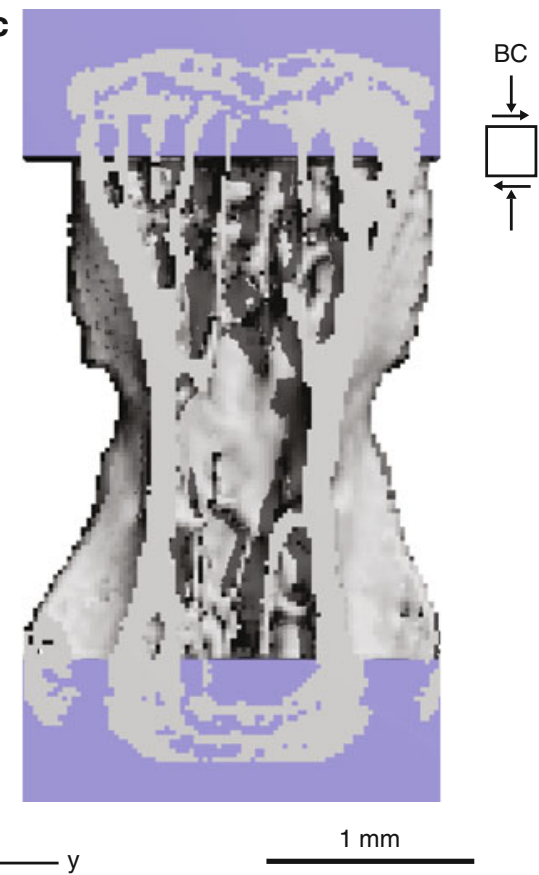

and the solution is determined using linear least square with the unconstrained subset of variables (passive set).

\subsection{Test models}

The procedure described above was first tested relative to artificially generated grid models, for which the loading directions that would cause homogeneous tissue loading can easily be anticipated, and second relative to results from an animal model, in which the loading history was well defined.

\subsubsection{Grid models}

The grid models consisted of a regular 3D grid of a large number of orthogonal bars in a cubic volume of $8 \mathrm{~mm}$. The spacing of the bars was $800 \mu \mathrm{m}$, and their thickness was $200 \mu \mathrm{m}$, thus mimicking typical morphometric properties of trabecular bone. The size of the voxels was $50 \mu \mathrm{m}$ isotropic. In a first model, all rods were aligned to the coordinate systems (Fig. 2a), whereas in a second model, rods were rotated by 45 degrees around the $z$-axis (Fig. 2b).

These models were converted to micro-FE models using the voxel conversion technique. Isotropic linear elastic material properties with a Young's modulus of 10 GPa (Ashman and Rho 1988; Bevill et al. 2009; Pressel et al. 2005; van Rietbergen et al. 1995; Zysset et al. 1999) and a Poisson's ratio of 0.3 (Lim and Hong 2000; Wang et al. 2009) were chosen according to commonly referred values for bone.

For each model, six different unit load cases were defined. The first three load cases represented a distributed normal force of $1 \mathrm{~N}$ in each of the three orthogonal directions; the last three load cases represented a distributed shear force in the three orthogonal directions (Fig. 2a,b). The micro-FE models were solved for each of these six load cases.

Model creation and micro-FE analysis were performed using Image Processing Language (IPL, Scanco Medical AG, Brüttisellen, Switzerland).

\subsection{Murine caudal vertebra models}

Previously generated data from an in vivo loading experiment are used in this study. This animal experiment is described in detail elsewhere (Lambers et al. 2009; Schulte et al. 2011). In short: fifteen-week-old female C57BL/6 mice underwent axial compressive loading of the sixth caudal vertebrae at either $8 \mathrm{~N}$ (loaded group; $n=8$ ) or $0 \mathrm{~N}$ (control group; $n=8$ ) for 3,000 cycles at $10 \mathrm{~Hz}$ three times per week for four weeks and weekly in vivo micro-computed tomography (micro-CT) scans. Loading was applied through pins inserted in adjacent vertebrae using a recently developed caudal vertebra axial compression device (CVAD) (Webster et al. 2008, 2010). During the remaining period, mice were able to freely move their tail. In vivo micro-CT scans were performed at $10.5 \mu \mathrm{m}$ resolution (viva-CT 40, Scanco Medical AG, Brüttisellen, Switzerland), and common bone morphometric parameters were determined.

For the present study, the in vivo micro-CT scans of the vertebrae at week 0,2 , and 4 were scaled down to a voxel size of $26 \mu \mathrm{m}$ and the bone phase was segmented. A voxel size of $26 \mu \mathrm{m}$ allowed micro-FE analysis in reasonable time (1 day) while still sufficiently capturing individual trabeculae of the mice vertebra with a thickness of $80 \mu \mathrm{m}$. At each end of the vertebra, a cartilaginous region was added using IPL to represent the intervertebral disks and the growth plate (Fig. 2c). 
Each voxel of the micro-CT image was then transformed into an equally sized brick element of the micro-FE model using the voxel conversion procedure. Material properties were chosen isotropic linear elastic, with a Young's modulus of $10 \mathrm{GPa}$ for the bone and $10 \mathrm{MPa}$ (Elliott and Sarver 2004) for the cartilaginous region. Both materials were assigned to a Poisson's ratio of 0.3 .

A total of six unit load cases were defined. These load cases represent distributed unit forces (prescribed total force of $1 \mathrm{~N}$ ) and distributed unit moments (prescribed total moment of $1 \mathrm{Nmm}$ ) in the three orthogonal directions (Fig. 2c). With all load cases, forces were applied to the ends of the intervertebral disks only.

\subsection{Homogeneity analysis}

To quantify the initial and final tissue SED homogeneity, the coefficient of variation $(\mathrm{CV}=\mathrm{SD} /$ mean) of the tissue SED distribution for both grid models and both mice groups at three measurement points was calculated.

\subsection{Statistical analysis}

For the mice vertebrae, each estimated scaling factor and CV were analyzed with a two-way analysis of variance (ANOVA) with repeated measures to test whether there was a significant effect of loading and time. The assumption of sphericity of the ANOVA with repeated measures was verified using Mauchly's test, and Bonferroni post hoc test was applied for pairwise comparison. If the assumption of sphericity was not met, Huynh-Feldt correction method was used. Further, a multivariate analysis of variance (MANOVA) followed by Bonferroni post hoc test was conducted to derive differences between the control and loaded group at each time point. The assumption of homogeneity of variances was verified using Levene's test. For all statistical analyses, IBM SPSS Statistics 19 (SPSS Inc., Chicago IL, USA) was used and a value of $p<0.05$ was considered as significant.

\section{Results}

\subsection{Grid models}

For both grid models, predominant forces were predicted in the direction of the rods. In the grid that was aligned with the coordinate system, equal scaling factors were determined for all normal forces $\left(s_{x}=s_{y}=s_{z}=2,479\right)$ while scaling factors for the shear forces were zero. For the rotated grid, relatively large scaling factors were determined for the normal forces $\left(s_{x}=197, s_{y}=186, s_{z}=1,963\right)$ and for the scaling factor of the shear force in the xy plane where the grid rods are aligned by $45^{\circ}$ to each other $\left(s_{x y}=1,538\right)$, whereas scaling factors for the other shear forces were zero. These force estimations reflect what we expected, since the applied forces in the direction of the grid rods should cause a uniform loading throughout the structure. Very little remaining inhomogeneity was found in both models: the aligned $\left(\mathrm{CV}_{\mathrm{a}}=9 \%\right)$ and the rotated $\left(C V_{\mathrm{r}}=16 \%\right)$ grid.

\subsection{Murine caudal vertebra models}

For the animal experiment, the scaling factor for the compressive force $s_{z}$ was much larger than for the other forces at any time point, thus indicating that compression should be the main loading mode in order to reach a homogeneous SED distribution. At the start of the experiment, a very similar mean scaling factor of about $s_{z}=16.0$ was predicted for both groups. After 4 weeks, however, the algorithm predicted a mean scaling factor of $s_{z}=29.7$ for the group that had the vertebrae not loaded ( $0 \mathrm{~N}$, control group) and $s_{z}=66.8$ for the group that had the vertebrae loaded ( $8 \mathrm{~N}$, loaded group) and these values were significantly different $(p=0.001)$. There was also a significant difference after 2 weeks $(p=0.038)$, whereas no significant effect of loading and time was found for the shear force scaling factors. Scaling factors for the torsion and bending load cases were very similar to each other and hardly changed over time. No significant effect of loading as well as of time was found.

If we assume that compressive loading is always the dominant loading mode (i.e., also in the natural situation before the experiment started), one can assume that $m_{z}=m_{\text {tot }}$ at all time points, and using Eq. 5, it is then possible to calculate the predicted force magnitudes as the square root of the scaling factor: $\alpha_{z}=4.21 \mathrm{~N}$ (mean) at $t=0$ and $\alpha_{z}=8.17 \mathrm{~N}$ (mean) at $t=4$ weeks for the loaded group (Fig. 3 and Table 1). For the control group, a value of $\alpha_{z}=3.90 \mathrm{~N}$ (mean) was found at $t=0$ and a value of $\alpha_{z}=5.45 \mathrm{~N}$ (mean) at $t=4$ weeks, which is much less than that of the loaded group but higher than the value at $t=0$, suggesting that the bone is getting slightly stronger and more aligned in the normal direction of physiological loading.

High remaining SED inhomogeneity of $\mathrm{CV}_{\mathrm{c}}=67.12 \%$ (mean) for the control group and $\mathrm{CV}_{1}=68.69 \%$ (mean) for the loaded group after 4-week loading was calculated when considering all scaled load cases together (Fig. 4 and Table 1). CV values decreased significantly ( $p<0.001)$ with scaling and time (Table 1), whereas no significant effect of loading was found.

\section{Discussion}

In this study, we introduced a novel approach to estimate external loading conditions based on bone morphology and tested it relative to artificially created models and relative 

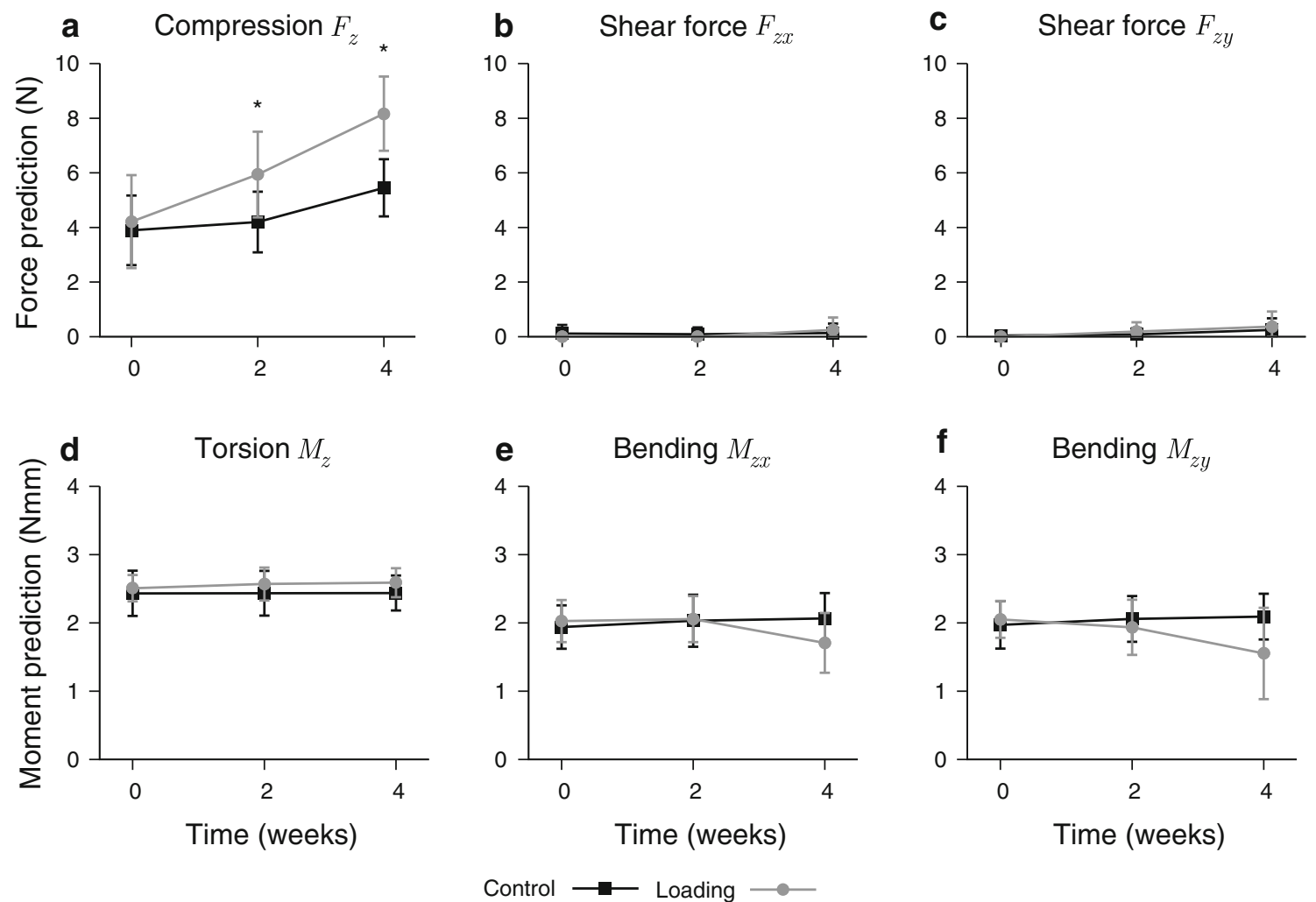

Fig. 3 Bone loading estimation for the murine caudal vertebrae that were loaded in vivo by either $8 \mathrm{~N}$ (loading group) or $0 \mathrm{~N}$ (control group), respectively. The algorithm predicted a significantly different

compressive force after 2 and 4 weeks for the two groups whereas other forces and moments were not different and hardly changed over time. ${ }^{*} p<0.05$; values refer to as mean $\pm \mathrm{SD}, n=8$ /group

Table 1 Bone loading estimations and coefficient of variation $(\mathrm{CV})$ for the murine caudal vertebrae that were loaded in vivo by either $8 \mathrm{~N}$ (loading group) or $0 \mathrm{~N}$ (control group), respectively at week 0,2 , and 4

\begin{tabular}{|c|c|c|c|c|c|c|}
\hline & \multicolumn{3}{|c|}{ Control group $(n=8)$} & \multicolumn{3}{|c|}{ Loading group $(n=8)$} \\
\hline & Week 0 & Week 2 & Week 4 & Week 0 & Week 2 & Week 4 \\
\hline$F_{z}(\mathrm{~N})$ & $3.90(1.36)$ & $3.94(1.01)$ & $5.45(1.12)$ & $4.21(1.82)$ & $5.94(1.67)$ & $8.17(1.45)$ \\
\hline$F_{z x}(\mathrm{~N})$ & $0.12(0.33)$ & $0.11(0.28)$ & $0.13(0.37)$ & $0.00(0.00)$ & $0.00(0.00)$ & $0.25(0.49)$ \\
\hline$F_{z y}(\mathrm{~N})$ & $0.04(0.12)$ & $0.10(0.26)$ & $0.25(0.46)$ & $0.00(0.00)$ & $0.19(0.36)$ & $0.37(0.59)$ \\
\hline$M_{z}(\mathrm{~N} \mathrm{~mm})$ & $2.43(0.35)$ & $2.46(0.37)$ & $2.44(0.27)$ & $2.51(0.21)$ & $2.57(0.26)$ & $2.59(0.23)$ \\
\hline$M_{z x}(\mathrm{~N} \mathrm{~mm})$ & $1.94(0.34)$ & $2.12(0.35)$ & $2.07(0.40)$ & $2.03(0.33)$ & $2.06(0.36)$ & $1.71(0.47)$ \\
\hline$M_{z y}(\mathrm{~N} \mathrm{~mm})$ & $1.97(0.37)$ & $2.12(0.34)$ & $2.09(0.36)$ & $2.05(0.29)$ & $1.94(0.43)$ & $1.55(0.71)$ \\
\hline $\mathrm{CV}_{\text {unscaled }}$ & $0.80(0.09)$ & $0.79(0.09)$ & $0.74(0.08)$ & $0.79(0.05)$ & $0.76(0.04)$ & $0.75(0.04)$ \\
\hline $\mathrm{CV}_{\text {scaled }}$ & $0.71(0.06)$ & $0.70(0.07)$ & $0.67(0.05)$ & $0.70(0.03)$ & $0.70(0.04)$ & $0.69(0.04)$ \\
\hline
\end{tabular}

Values refer to as mean (SD)

to results of an animal experiment in which murine caudal vertebrae received additional high compressive loading in one group and no additional loading in a control group. The results show that the method was able to correctly determine loading conditions for the test models and to identify a significant difference in loading history between the loaded and control groups, with, for the loaded group, an increase in calculated load magnitude over time reaching an end value that was well in agreement with the load applied in the experiment.

The fact that the calculated forces increased over time for the loaded group indicates that the force that was applied during the loading period was higher than the normal physiological load and that this led to bone adaptation. This is confirmed by an increase in the total bone volume (BV) of the vertebrae on average by $13.1 \%$ during the loading period. 
Fig. 4 Tissue SED distributions for the murine caudal vertebrae that were loaded in vivo by either $8 \mathrm{~N}$ (loading group) or $0 \mathrm{~N}$ (control group), respectively, at week 4. Both groups showed a high remaining inhomogeneity in tissue SED

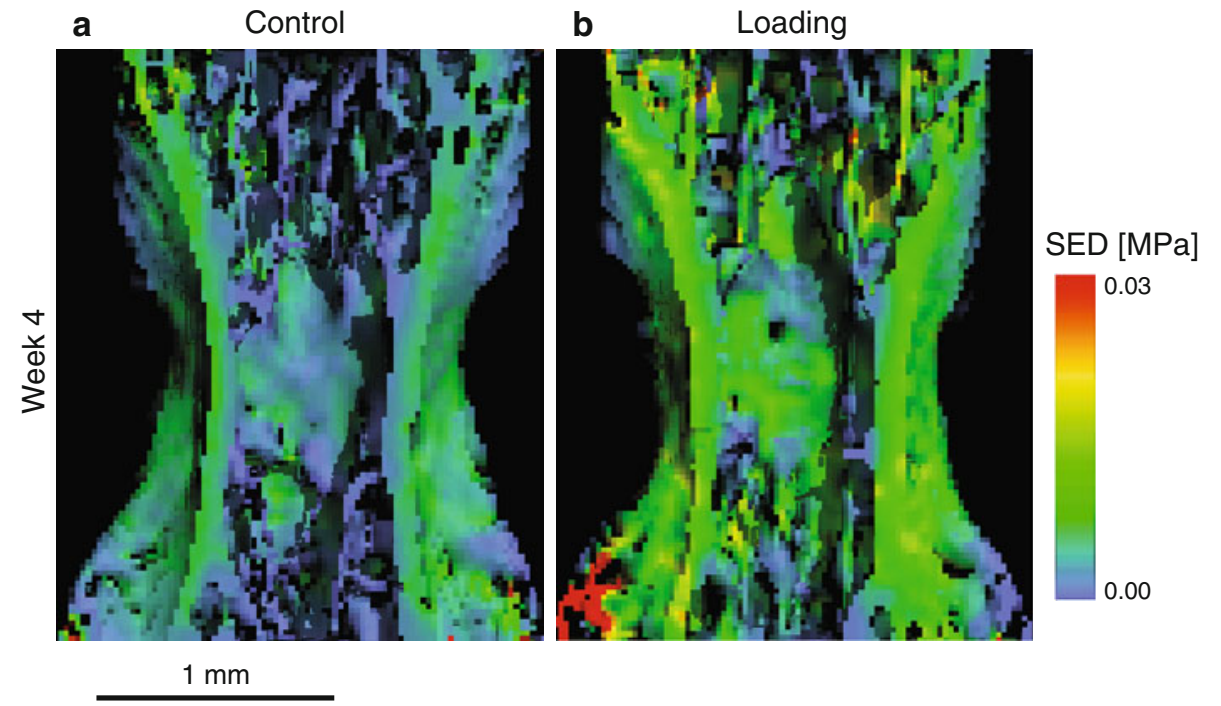

Unexpectedly, there was also a trend toward an increase in calculated force for the control group. This increase was found to relate to a small increase in BV of $6.7 \%$ (mean), indicating that the animals were still growing. This was confirmed by the fact that the vertebrae slightly increased in length by $1.2 \%$ (mean) during the experiment.

To investigate to what extent homogeneity of bone tissue loading can be achieved when using a limited set of 'plausible' external forces, the coefficient of variation (CV) of the scaled strain-energy density (SED) distribution in the mice vertebrae was calculated. A considerable inhomogeneity of tissue SED of about $67 \%$ for the control group and $69 \%$ for the loaded group remained. In a previous study, using density-based bone loading estimations, Kenneth J. Fischer (personal communication) found only a final tissue loading inhomogeneity of about $20 \%$. This difference between studies, however, likely relates to the fact that the density-based approach accounts for less detail compared to our method. Because no uniform tissue loading was found, not even when using an optimization algorithm, it could be that more diverse load cases need to be included, for example load cases representing forces exerted by ligaments or muscles on the processes. Another possible explanation would be that a 'lazy zone' or 'dead zone' (Carter 1982; Cowin 1987; Frost 1964, 1997; Huiskes et al. 1987) exists in bone cell mechanosensitivity where bone cells do not response to the mechanical stimulus as long as the signal is within a certain range. In previous bone remodeling studies, a rather big 'lazy zone' was required to achieve more realistic results (Huiskes et al. 1992), which is also in agreement with the high inhomogeneity we found in our study. Finally, it is possible that genetic or other factors play an important role when the bone structure develops and that bone in fact is only partially optimized for loading conditions (Jepsen 2009; Karasik and Kiel 2010). For example, if bone growth also takes place in the absence of loading, only part of the structure will be load adapted. If this indeed is an important factor, better results would be expected for bone in older subjects that might be more adapted to their loading history. But also in fully grown bone, other factors of biological nature like calcium homeostasis or sex hormones could be physiologically more important than a bone structure perfectly adapted to its loading regime (Beaupre et al. 1990; Frost 1987; Harada and Rodan 2003; Manolagas 2000; Robling et al. 2006).

We expected that the $\mathrm{CV}$ values for the animals in the loaded group would reduce over time since an adaptation process where bone apposition occurs at highly loaded locations and bone resorption at lower-loaded locations reduces the inhomogeneity. We also expected that in the control group the inhomogeneity of the SED distribution would stay constant, since very little changes in bone adaptation are expected. The results of the analyses, however, show only a minor decrease in CV values over time for both groups. Nevertheless, plots of the SED distribution in the vertebrae clearly show a reduction in peak SED values when comparing baseline with 4 weeks. This effect is more pronounced for the loaded than for the control group (Fig. 5). This is not well reflected by the $\mathrm{CV}$ values since the reduction in these peak values has only a minor effect on the calculated standard deviation of the distribution.

In the mice vertebrae, most of the loading is transmitted from the intervertebral disk to the vertebrae. This allows applying unit forces and moments in all possible directions to the disks and thus capturing the possible physiological loading conditions with a relatively small number of load cases. However, in more complex situations, e.g., the proximal femur, unit loads have to be defined in a different way. Since forces act perpendicular to the articular surface, a large number of unit forces could be evenly distributed over the surface in complex joints. This also captures the range of 
Fig. 5 Tissue SED distribution of the compression load case for the murine caudal vertebrae that were loaded in vivo by either $8 \mathrm{~N}$ (loading group) or $0 \mathrm{~N}$ (control group), respectively, at weeks 0 and 4 . Peak SED values were effectively reduced when comparing baseline and week 4 in the loaded group, whereas the reduction was less pronounced in the control group

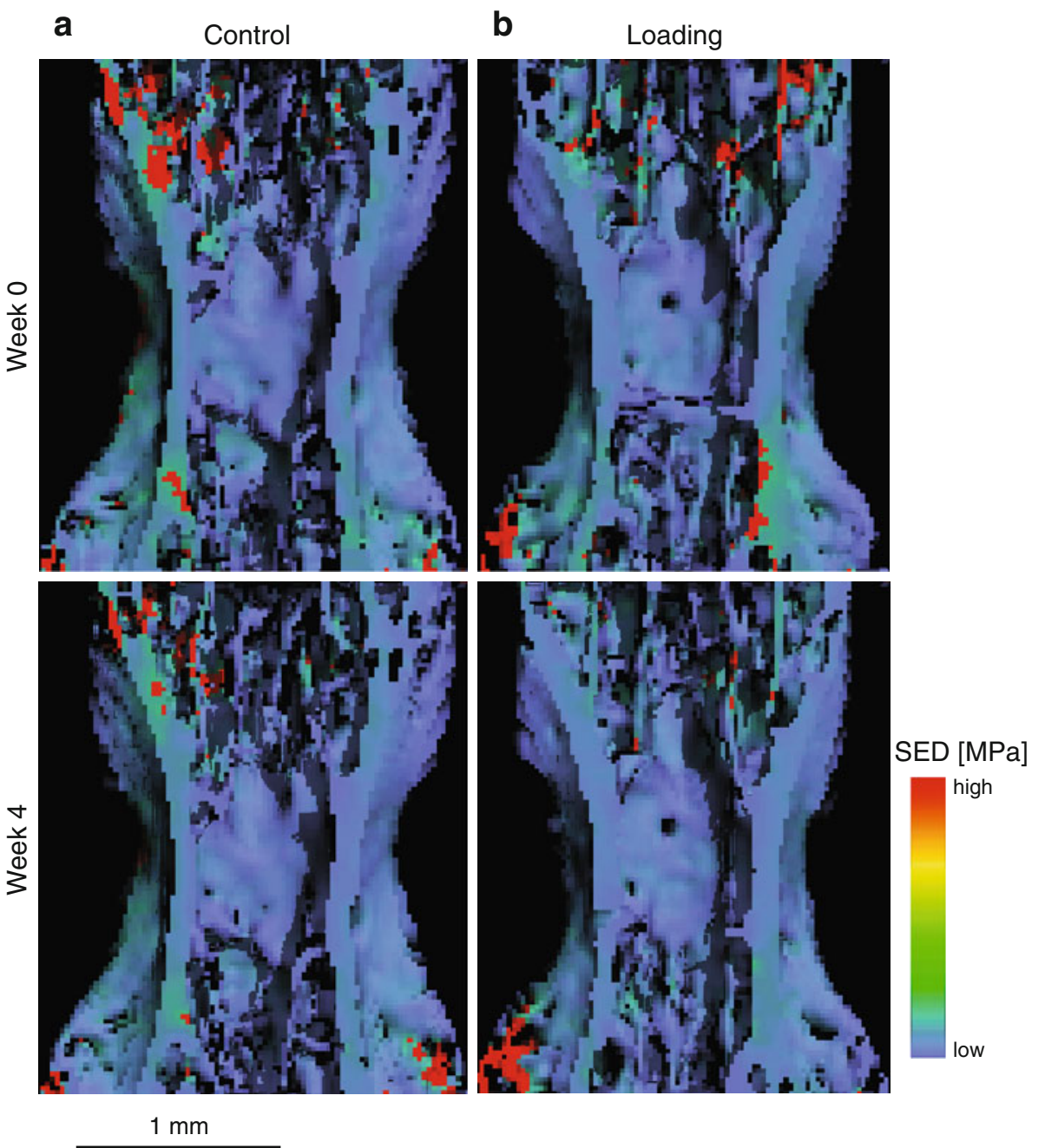

possible physiological loading but also causes a much higher number of unit loads than six, as used here in the vertebrae. Increasing the number of unit loads also increases the number of combinations that lead to a uniform loading and therefore might lead to unexpected load predictions.

There are some limitations to our study that have to be considered. We assume that bone adapts to its internal architecture in order to achieve a uniform tissue loading that we quantified as SED. Several other candidates for the mechanical signal have been proposed, such as fluid flow shear stress (Burger and Klein-Nulend 1999; Burra and Jiang 2009; Rath et al. 2010) or micro-damage (Mori and Burr 1993; Robling et al. 2006). However, earlier studies have demonstrated that the choice of the mechanical signal is not very critical since most of these signals are highly correlated (Ruimerman et al. 2005). Another limitation is that the unit load cases have to be preselected according to the loading conditions in the physiological situation of the bone. Defining these loads and applying them in micro-FE models can be difficult, and often the physiological situation has to be simplified.

In conclusion, we found that the bone loading estimation algorithm introduced here can provide information about the loading history. However, more work will be needed to establish the accuracy and sensitivity of the procedure and to find out what part of the bone structure is caused by adaptation and what part is due to other factors. For the purpose of bone remodeling simulation studies, however, this might be of lesser importance since in this case the response to changes in the loading is of interest rather than the actual loading history.

Acknowledgments Funding from the European Union for the osteoporotic virtual physiological human project (VPHOP FP7-ICT2008223865 ) is gratefully acknowledged.

Open Access This article is distributed under the terms of the Creative Commons Attribution Noncommercial License which permits any 
noncommercial use, distribution, and reproduction in any medium, provided the original author(s) and source are credited.

\section{References}

Abel R, Macho GA (2011) Ontogenetic changes in the internal and external morphology of the ilium in modern humans. J Anatomy 218(3):324-335. doi:10.1111/j.1469-7580.2011.01342.x

Adams DJ, Spirt AA, Brown TD, Fritton SP, Rubin CT, Brand RA (1997) Testing the daily stress stimulus theory of bone adaptation with natural and experimentally controlled strain histories. J Biomech 30(7):671-678

Ashman RB, Rho JY (1988) Elastic modulus of trabecular bone material. J Biomech 21(3):177-181

Beaupre GS, Orr TE, Carter DR (1990) An approach for time-dependent bone modeling and remodeling-application: a preliminary remodeling simulation. J Orthop Res 8(5):662-670. doi:10.1002/ jor. 1100080507

Bevill G, Eswaran SK, Farahmand F, Keaveny TM (2009) The influence of boundary conditions and loading mode on high-resolution finite element-computed trabecular tissue properties. Bone 44(4): 573-578. doi:10.1016/j.bone.2008.11.015

Bona MA, Martin LD, Fischer KJ (2006) A contact algorithm for density-based load estimation. J Biomech 39(4):636-644. doi:10. 1016/j.jbiomech.2005.01.006

Burger EH, Klein-Nulend J (1999) Mechanotransduction in bone-role of the lacuno-canalicular network. FASEB J 13(Suppl):S101-112

Burra S, Jiang JX (2009) Connexin 43 hemichannel opening associated with Prostaglandin E(2) release is adaptively regulated by mechanical stimulation. Commun Integr Biol 2(3):239-240

Carter DR (1982) The relationship between in vivo strains and cortical bone remodeling. Crit Rev Biomed Eng 8(1):1-28

Cowin SC (1987) Bone remodeling of diaphyseal surfaces by torsional loads: theoretical predictions. J Biomech 20(11-12):11111120

Ding M, Odgaard A, Danielsen CC, Hvid I (2002) Mutual associations among microstructural, physical and mechanical properties of human cancellous bone. J Bone Joint Surg Br 84(6):900-907

Elliott DM, Sarver JJ (2004) Young investigator award winner: validation of the mouse and rat disc as mechanical models of the human lumbar disc. Spine Phil Pa (1976)29(7):713-722

Fischer KJ, Jacobs CR, Carter DR (1995) Computational method for determination of bone and joint loads using bone density distributions. J Biomech 28(9):1127-1135

Fischer KJ, Jacobs CR, Levenston ME, Cody DD, Carter DR (1998) Bone load estimation for the proximal femur using single energy quantitative CT data. Comput Methods Biomech Biomed Eng 1(3):233-245

Fischer KJ, Jacobs CR, Levenston ME, Cody DD, Carter DR (1999) Proximal femoral density patterns are consistent with bicentric joint loads. Comput Methods Biomech Biomed Eng 2(4): 271-283

Forwood MR, Turner CH (1995) Skeletal adaptations to mechanical usage: results from tibial loading studies in rats. Bone 17(Suppl 4): 197S-205S

Frost HM (1964) The laws of bone structure

Frost HM (1987) Bone "mass" and the "mechanostat": a proposal. Anat Rec 219(1):1-9. doi:10.1002/ar.1092190104

Frost HM (1997) On our age-related bone loss: insights from a new paradigm. J Bone Miner Res 12(10):1539-1546. doi:10.1359/jbmr. 1997.12.10.1539
Giesen EB, van Eijden TM (2000) The three-dimensional cancellous bone architecture of the human mandibular condyle. J Dent Res 79(4):957-963

Goldstein SA, Matthews LS, Kuhn JL, Hollister SJ (1991) Trabecular bone remodeling: an experimental model. J Biomech 24(Suppl 1): $135-150$

Harada S, Rodan GA (2003) Control of osteoblast function and regulation of bone mass. Nature 423(6937):349-355. doi:10.1038/ nature 01660

Hildebrand T, Laib A, Muller R, Dequeker J, Ruegsegger P (1999) Direct three-dimensional morphometric analysis of human cancellous bone: microstructural data from spine, femur, iliac crest, and calcaneus. J Bone Miner Res 14(7):1167-1174. doi:10.1359/ jbmr.1999.14.7.1167

Huiskes R, Weinans H, Grootenboer HJ, Dalstra M, Fudala B, Slooff TJ (1987) Adaptive bone-remodeling theory applied to prostheticdesign analysis. J Biomech 20(11-12):1135-1150

Huiskes R, Weinans H, van Rietbergen B (1992) The relationship between stress shielding and bone resorption around total hip stems and the effects of flexible materials. Clin Orthop Relat Res 274:124-134

Jepsen KJ (2009) Systems analysis of bone. Wiley Interdiscip Rev Syst Biol Med 1(1):73-88. doi:10.1002/wsbm.15

Karasik D, Kiel DP (2010) Evidence for pleiotropic factors in genetics of the musculoskeletal system. Bone 46(5):1226-1237. doi:10. 1016/j.bone.2010.01.382

Lambers FM, Kuhn G, Gerhard FA, Müller R (2009) Load induced bone adaptation monitored with in vivo micro-computed tomography. In: Book of abstracts ICCB 2009, IV international congress on computational bioengineering, Bertinoro, Italy, ISSN 2036-9247 (http://www.iccb2009.org/abstracts), September 16-18, p 139

Lawson CL, Hanson RJ (1974) Solving least squares problems. Prentice-Hall, Englewood Cliffs

Lim TH, Hong JH (2000) Poroelastic properties of bovine vertebral trabecular bone. J Orthop Res 18(4):671-677. doi:10.1002/jor. 1100180421

Manolagas SC (2000) Birth and death of bone cells: basic regulatory mechanisms and implications for the pathogenesis and treatment of osteoporosis. Endocr Rev 21(2):115-137

Mori S, Burr DB (1993) Increased intracortical remodeling following fatigue damage. Bone 14(2):103-109

Mullender MG, Huiskes R (1995) Proposal for the regulatory mechanism of Wolff's law. J Orthop Res 13(4):503-512. doi:10.1002/ jor.1100130405

Pressel T, Bouguecha A, Vogt U, Meyer-Lindenberg A, Behrens BA, Nolte I, Windhagen $\mathrm{H}$ (2005) Mechanical properties of femoral trabecular bone in dogs. Biomed Eng Online 4(1):17. doi:10.1186/ 1475-925X-4- 17

Rath AL, Bonewald LF, Ling J, Jiang JX, Van Dyke ME, Nicolella DP (2010) Correlation of cell strain in single osteocytes with intracellular calcium, but not intracellular nitric oxide, in response to fluid flow. J Biomech 43(8):1560-1564. doi:10.1016/ j.jbiomech.2010.01.030

Robling AG, Castillo AB, Turner CH (2006) Biomechanical and molecular regulation of bone remodeling. Annu Rev Biomed Eng 8:455498. doi:10.1146/annurev.bioeng.8.061505.095721

Rubin CT, Lanyon LE (1987) Kappa Delta Award paper. Osteoregulatory nature of mechanical stimuli: function as a determinant for adaptive remodeling in bone. J Orthop Res Offic Publ Orthop Res Soc 5(2):300-310. doi:10.1002/jor.1100050217

Ruimerman R, Van Rietbergen B, Hilbers P, Huiskes R (2005) The effects of trabecular-bone loading variables on the surface signaling potential for bone remodeling and adaptation. Ann Biomed Eng 33(1):71-78 
Schulte FA, Lambers FM, Kuhn G, Muller R (2011) In vivo micro-computed tomography allows direct three-dimensional quantification of both bone formation and bone resorption parameters using timelapsed imaging. Bone 48(3):433-442. doi:10.1016/j.bone.2010. 10.007

van Rietbergen B, Weinans H, Huiskes R, Odgaard A (1995) A new method to determine trabecular bone elastic properties and loading using micromechanical finite-element models. J Biomech 28(1):69-81

Wang Q, Xie H, Tang P, Yao Q, Huang P, Chen P, Huang F (2009) A study on the mechanical properties of beagle femoral head using the digital speckle correlation method. Med Eng Phys 31(10):1228-1234. doi:10.1016/j.medengphy.2009.07.021

Webster D, Wasserman E, Ehrbar M, Weber F, Bab I, Muller R (2010) Mechanical loading of mouse caudal vertebrae increases trabecular and cortical bone mass-dependence on dose and genotype. Biomech Model Mechanobiol 9(6):737-747. doi:10.1007/ s10237-010-0210-1
Webster DJ, Morley PL, van Lenthe GH, Muller R (2008) A novel in vivo mouse model for mechanically stimulated bone adaptation-a combined experimental and computational validation study. Comput Methods Biomech Biomed Eng 11(5):435-441. doi:10.1080/10255840802078014

Whitehouse WJ, Dyson ED (1974) Scanning electron microscope studies of trabecular bone in the proximal end of the human femur. J Anat 118(Pt 3):417-444

Wolff J (1892) Das Gesetz der Trasnformation der Knochen. Verlag von August Hirschwalden, Berlin

Zysset PK, Guo XE, Hoffler CE, Moore KE, Goldstein SA (1999) Elastic modulus and hardness of cortical and trabecular bone lamellae measured by nanoindentation in the human femur. J Biomech 32(10):1005-1012 